\title{
Application of IOT to Forest Management Taking Fushan Botanical Garden as An Example
}

\author{
Shuo-Tsung Chen ${ }^{1}$, Chih-Chiang $\mathrm{Hua}^{2}$, and Ching-Chun Chuang ${ }^{3 *}$ \\ ${ }^{1}$ Bachelor Program in Interdisciplinary, National Yunlin University of Science and Technology;123 University Road, \\ Section 3, Douliou, Yunlin 64002, Taiwan, R.O.C. \\ ${ }^{2}$ Department of Electrical Engineering, National Yunlin University of Science and Technology;123 University Road, \\ Section 3, Douliou, Yunlin 64002, Taiwan, R.O.C. \\ ${ }^{3}$ Department of Computer Science and Information Engineering, National Formosa University; No. 63, Wunhua Rd.,
} Huwei Toshnship, Yunlin 632, Taiwan, R.O.C.

E-mail:shough34@yahoo.com.tw,huacc@yuntech.edu.tw,austincc@nfu.edu.tw

\begin{abstract}
In recent years, the Internet of Things (IoT) technology has developed rapidly and has been successfully applied in different fields, and has begun to expand the application context of the Internet of Things. This work aims to study how to apply the IoT technology to forestry management, including: 1 . Forestry management using wireless network communication technology of Low Power Wide Area Network (LPWAN) such as LoRa and NB-IoT; 2. Apply different sensing technologies to survey resource of forest and monitor the microclimate changes in forest. In order to actually verify the proposed feasible communication technology, sensors, and sensor arrangement, we chose the Fushan Botanical Garden with the most diverse and complex terrain in Taiwan as the experimental site. We actually built LoRa and NB-IoT communication equipment (including repeat equipment) and various sensors to transmit the real-time sensing data. The returned data also verifies the successful operation of various communication devices and sensors.
\end{abstract}

Keywords: Internet of Things (IoT), Low Power Wide Area Network (LPWAN), LoRa, NB-IoT

\section{Introduction}

The application of IoT in forestry can be divided into three major aspects: forest environment and resource monitoring planning, forest fire intelligent management and illegal logging prevention. Environmental and resource monitoring should be a more successful part of the application of wireless sensing network (WSN) in the forestry Internet of Things. Zhang et al. [1] proposed a forest environmental factor collection platform based on the ZIGBEE protocol. This platform includes various terminal monitoring equipment such as temperature, humidity, water level, gas, micro-electromechanical system (mems), photoresistor (LDR) and human infrared sensor (PIR), which are all included in the platform's planning and application. Suciu [2] proposed a wireless sensor network architecture with a solution that effectively uses available resources; that is, it tries to optimize the duty cycle of a single sensor (but a single node) or maximize the service life of the network.

Kelvin Hirschet al. [3] proposed an ecosystem based on Canadian forest fire protection, as shown in Figure 7, which is a practical method of sustainable forest management. The goal is to use forest management practices in an active and planned way to reduce the total area burned by wildfires and the risks associated with the use of designated fires. The authors [4,5] proposed a forest fire detection system, which consists of sensor

(C) The 2021 International Conference on Artificial Life and Robotics (ICAROB2021), January 21 to 24, 2021 
nodes randomly deployed in the forest area. Each node is equipped with a temperature sensor. Nodes periodically check the environment to determine whether there is an emergency. When some sensor nodes detect a significant change in temperature, they will broadcast a data packet containing their measured value and present the data on the computer web page and mobile phone page. Suguvanam et al. [6] proposed a model to prevent the smuggling of valuable trees such as sandalwood and red sandalwood in forest areas. As shown in Figure 10, their model has three units: 1 . Tree unit 2. Sub Server Unit 3. Forest officer.

In this work, we implement the IoT technology for forestry management in Fushan Botanical Garden in Taiwan. In the areas with $3 \mathrm{G} / 4 \mathrm{G}$, we install NB-IoTsupport sensors and continuously send out their sensing data by using NB-IoT communication technology. Due to the fact that Fushan Botanical Garden has the most diverse and complex terrain without $3 \mathrm{G} / 4 \mathrm{G}$, the real-time sensing data including illuminance, atmospheric pressure, ultraviolet light, and carbon dioxide are repeated to two repeat stations which is installed on hillside in order. Thus, these sensing data are transmitted to LoRa getway connected with $3 \mathrm{G} / 4 \mathrm{G}$. Finally, the real-time sensing data are transmitted to web server.

The rest of this work are as follows. Section 2 proposes Forest Sensors and Communication Technology. Section 3 presents the implementation and experiment results. Section 4 lists conclusion.

\section{Proposed Forest Sensors and Communication Technology}

This section presents proposed forest sensors and communication technology for the Fukuyama Botanical Garden in Yilan County, New Taipei City, Taiwan. A few areas in the Fukuyama Botanical Garden have 3G/4G signals, but most areas do not have $3 \mathrm{G} / 4 \mathrm{G}$ signals. Therefore, as shown in Figure 1, we use two wireless network communication technologies of Low Power Wide Area Network (LPWAN), NB-IoT and LoRa, combined with their respective sensors to send the sensing data back to the Forestry Bureau of the Council of Agriculture of the Executive Yuan (Forestry Bureau). The detail is presented in subsections 2.1 and 2.2.

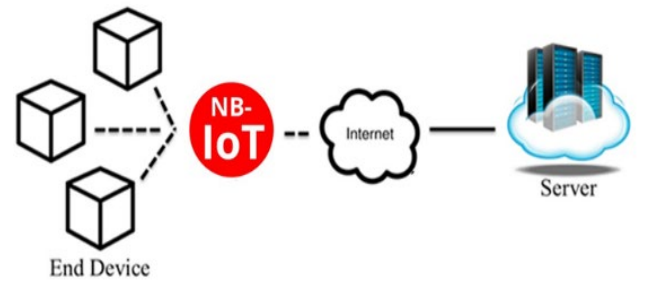

(a) NB-IoT communication technology and its respective sensors

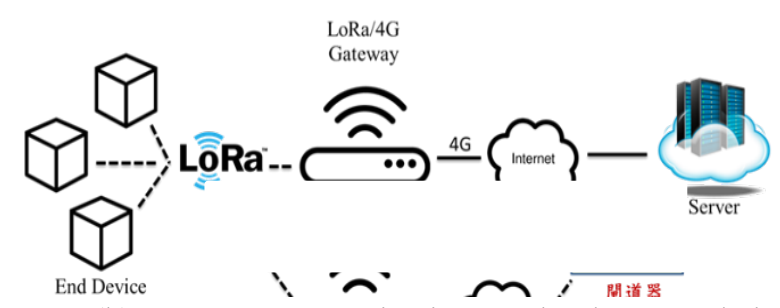

(b) LoRa communication technology and its respective sensors

Fig. 1. Two communication technologies, NB-IoT and LoRa, combined with their respective sensors.

\subsection{NB-IoT communication technology and its respective sensors}

In a few areas with $3 \mathrm{G} / 4 \mathrm{G}$, we use NB-IoT communication technology to continuously return the sensing data from NB-IoT-support sensors.

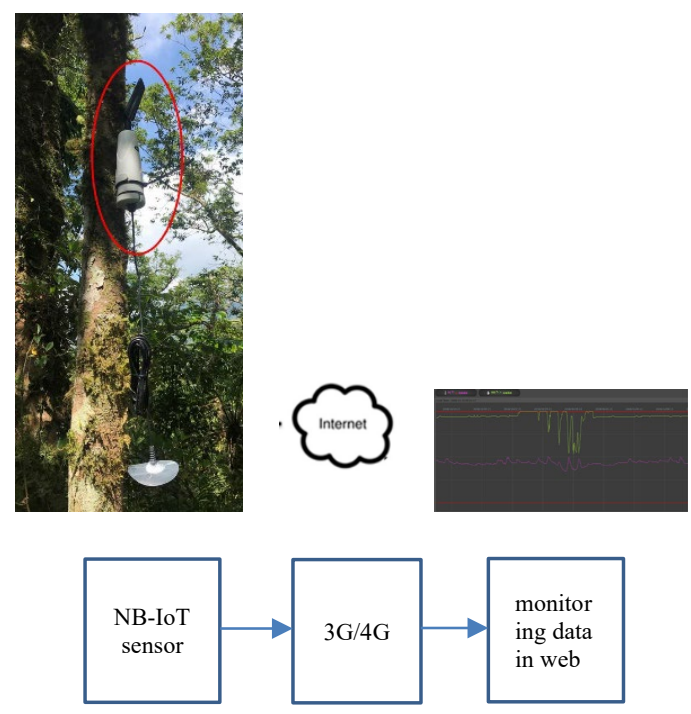

Fig. 2. Flowchart of NB-IoT method for returning the sensing data. 


\subsection{LoRa communication technology and its respective sensors}

Due to the complex and diverse terrain of the Fushan site and most areas have no $3 \mathrm{G} / 4 \mathrm{G}$ signal, the LoRa signal repeat method of this work will continuously return the sensing data of the LoRa-support solar micro-weather station.

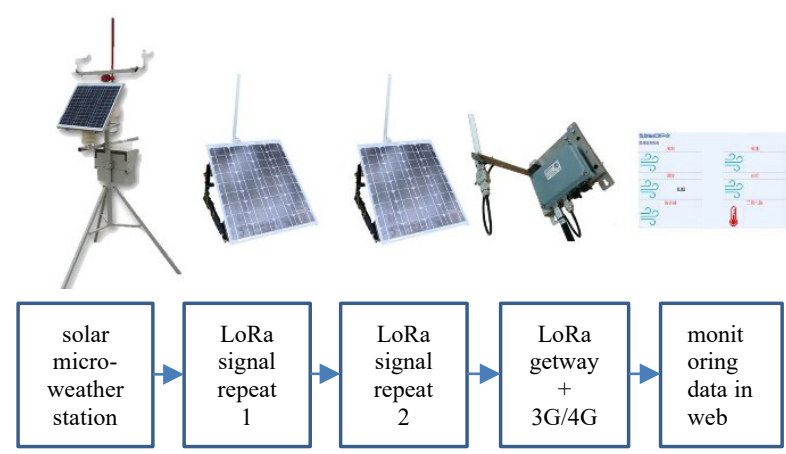

Fig. 3. Flowchart of LoRa signal repeat method for returning the sensing data of the LoRa-support solar micro-weather station.

\section{Implementation and Experiment results}

This section shows the implementation of the proposed IoT planning applied to forest management and its experimental results.

\subsection{Implementation and Experimental Results of NB-IoT Planning}

In the areas with $3 \mathrm{G} / 4 \mathrm{G}$, as shown in Figure 4, we first install NB-IoT-support sensors as shown in Figure 5 and continuously send out their sensing data by using NB-IoT communication technology. Next, the real-time sensing data including temperature and humidity are connected with $3 \mathrm{G} / 4 \mathrm{G}$ and finally transmitted to web server as shown in Figure 6.

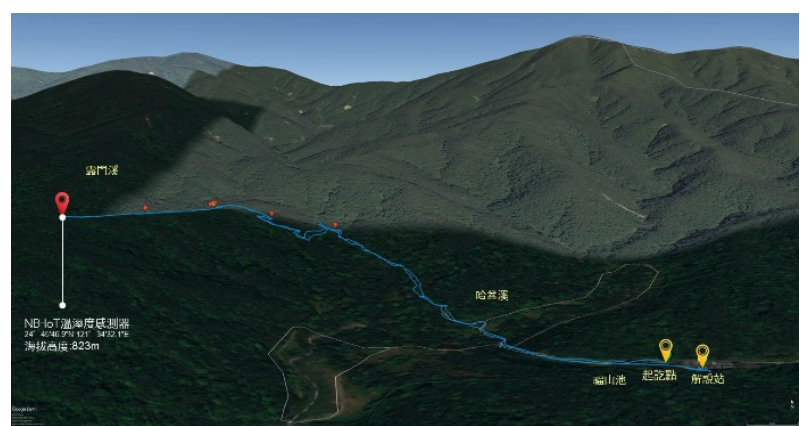

Fig. 4. Implementation of NB-IoT-support sensors and communication technology.

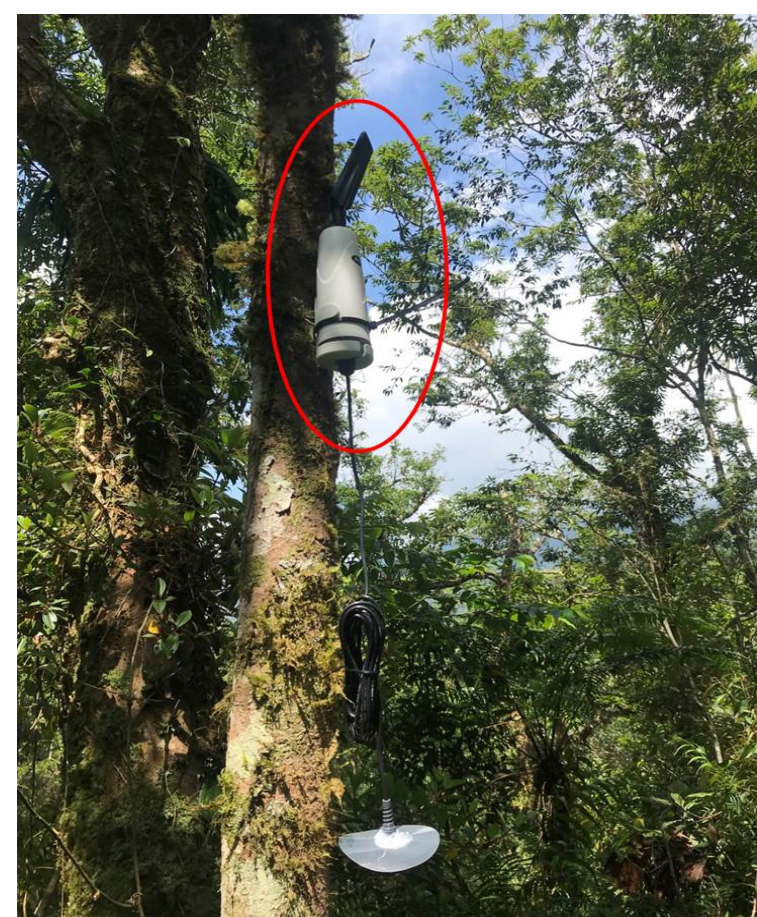

Fig. 5. NB-IoT-support temperature and humidity sensors.

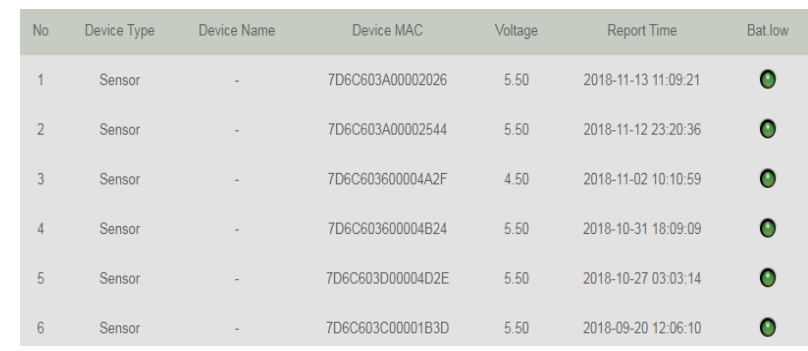

Fig. 6. Real-time data of temperature and humidity.

\subsection{Implementation and Experimental Results of LoRa Repeat Planning}

In the large areas without $3 \mathrm{G} / 4 \mathrm{G}$, we first install LoRasupport solar micro-weather station in the top of some mountain as shown in Figure 7 and continuously send out sensing data by using LoRa communication technology. Next, the real-time sensing data including illuminance, atmospheric pressure, ultraviolet light, and carbon dioxide are repeated to two repeat stations which is installed on hillside in order. Respectively, these sensing data are then transmitted to LoRa getway which is connected with $3 \mathrm{G} / 4 \mathrm{G}$. Finally, these real-time sensing data are transmitted to web server as shown in Figure 8.

(C) The 2021 International Conference on Artificial Life and Robotics (ICAROB2021), January 21 to 24, 2021 


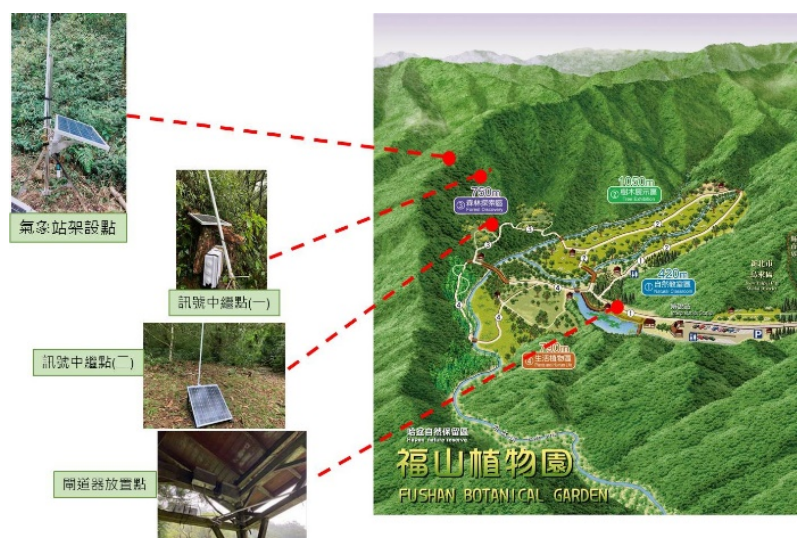

Fig. 7. Implementation of LoRa-support solar micro weather station, two repeat stations, and communication technology.

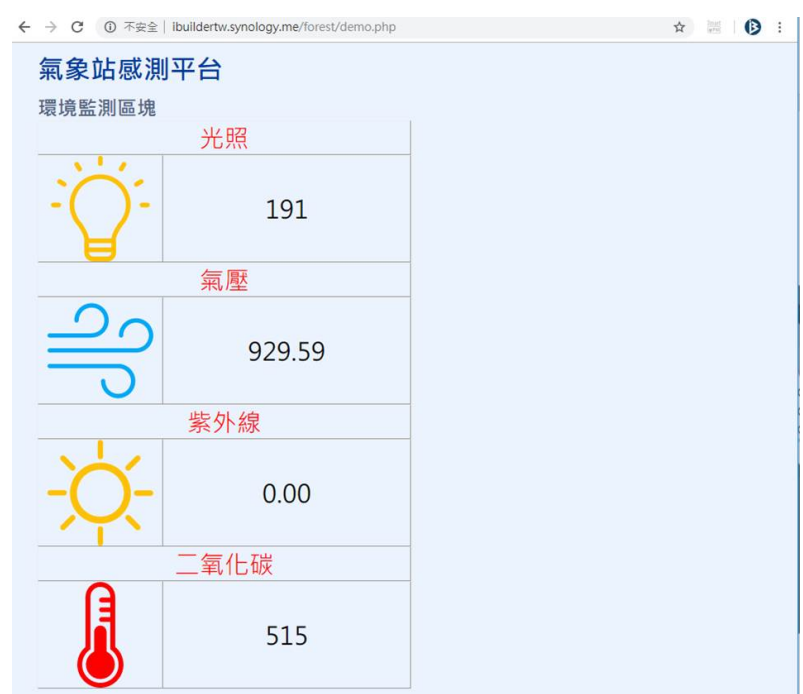

Fig. 8. Real-time data of illuminance, atmospheric pressure, ultraviolet light, carbon dioxide.

\section{Conclusions}

In this work, we study how to apply the IoT technology to forestry management in Fushan Botanical Garden in Taiwan. In the areas with $3 \mathrm{G} / 4 \mathrm{G}$, we install NB-IoTsupport sensors and continuously send out their sensing data by using NB-IoT communication technology. Due to the fact that Fushan Botanical Garden has the most diverse and complex terrain without $3 \mathrm{G} / 4 \mathrm{G}$, the real-time sensing data including illuminance, atmospheric pressure, ultraviolet light, and carbon dioxide are repeated to two repeat stations which is installed on hillside in order. Respectively, these sensing data are then successfully transmitted to LoRa getway which is connected with
3G/4G. Finally, these real-time sensing data are transmitted to web server.

\section{References}

1. Zhang Yu, Liu Xugang, Geng Xue, Li Dan, "IoT Forest Environmental Factors Collection Platform Based on ZIGBEE," CYBERNETICS AND INFORMATION TECHNOLOGIES, Volume 14, Special Issue, 2014, pp.51-62.

2. George Suciu, Ramona Ciuciuc, Adrian Pasat, and Andrei Scheianu, "Remote Sensing for Forest Environment Preservation," Advances in Intelligent Systems and Computing, 2017, DOI 10.1007/978-3-319-56538-5_23

3. Kelvin Hirsch, Victor Kafka, Cordy TJlmstra, Rob McAlpine', Brad Hawkes, Herman Stegehuis, Sherra Quintilio, Sylvie Gauthier, and Karl Peck, "Fire-smart forest management: A pragmatic approach to sustainable forest management in fire-dominated ecosystems," THE FORESTR Y CHRONICLE MARCWAPRIL, VOL. 77, NO.2, pp. 357-363, 2001

4. Pouya Bolourchi and Sener Uysa, "Forest Fire Detection with Wireless Sensor Networks," 2013 Fifth International Conference on Computational Intelligence, Communication Systems and Networks.

5. Murat Dener, Yunus Özkök, Cevat Bostancioglu, "Fire Detection Systems in Wireless Sensor Networks," World Conference on Technology, Innovation and Entrepreneurship, Social and Behavioral Sciences, 195 ( 2015 ) pp.1846-1850.

6. Suguvanam K, Senthil Kumar, Partha Sarathy, Karthick, Raj Kumar, "Innovative Protection of Valuable Trees from Smuggling Using RFID and Sensors," International Journal of Innovative Research in Science, Engineering, and Technology, Vol. 6, Issue 3, March 2017, pp.38363845. 